\title{
PROPER EFFICIENCY IN LINEAR VECTOR MAXIMUM PROBLEMS WITH NONLINEAR CONSTRAINTS
}

\author{
T. R. GULATI and M. A. ISLAM
}

(Received 6 April 1987)

Communicated by B. Mond

\begin{abstract}
A linear vector maximum problem with nonlinear constraints is considered. A condition is derived which is necessary for an efficient solution and sufficient for a properly efficient solution of this problem. This leads to sufficient conditions for an efficient solution to be properly efficient. An example is discussed at the end.
\end{abstract}

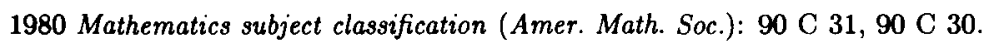

\section{Introduction}

A vector maximum problem arises when two or more functions are to be maximized over a feasible region. Generally, the concept of optimal solution does not work in vector maximum problems. Therefore, a more general concept of efficient solution is adopted. To eliminate the undesirable anomaly of efficient solutions, Kuhn and Tucker [9] proposed the concept of proper efficiency. Klinger [8] observed that every improperly efficient solution has this anomaly. Geoffrion [4] discussed an example wherein a solution proper in the sense of Kuhn and Tucker demonstrates a similar anomaly. To exclude all such undesirable solutions, Geoffrion [4] reformulated the definition of a properly efficient solution. Using this definition Isermann [5] for linear vector maximum problem and Choo [3] for linear fractional vector maximum problem with linear constraints proved

(C) 1989 Australian Mathematical Society $0263-6115 / 89 \$ A 2.00+0.00$ 
that every efficient solution is properly efficient. Necessary conditions for efficient solution of vector maximum problems with fractional objectives and linear constraints have been obtained by Kaul and Gupta [6], [7] and Bhatia and Gupta [2]. Benson and Morin [1] derived necessary and sufficient conditions for an efficient solution to be properly efficient for vector maximum problems with concave objectives and convex feasible region. However, their conditions involve stability of certain problems. Here we consider a linear vector maximum problem with nonlinear constraints and derive a condition which is necessary for an efficient solution and sufficient for a properly efficient solution.

\section{The problem and preliminaries}

We consider the following vector maximum problem:

$$
\begin{gathered}
\operatorname{maximize} C x=\left[c_{1} x, c_{2} x, \ldots, c_{k} x\right]^{T} \\
\text { subject to } x \in X=\left\{x \in R^{n}: g(x) \leqq 0\right\}
\end{gathered}
$$

where $c_{i} \in R^{n}$ for each $i \in K=\{1,2, \ldots, k\}, C$ is a $k \times n$ matrix and $g: R^{n} \rightarrow$ $R^{m}$ is a differentiable function.

The following convention for inequalities will be used. If $x, y \in R^{n}$, then $x \geqq y \Leftrightarrow x_{i} \geqq y_{i}, i=1,2, \ldots, n ; x \geqq y \Leftrightarrow x \geqq y$ and $x \neq y ; x>y \Leftrightarrow x_{i}>y_{i}$, $i=1,2, \ldots, n$. Also, for $x^{0} \in X, \bar{I}=\left\{i: g_{i}\left(x^{0}\right)=0\right\}$ and $g_{I}$ will denote the active constraints at $x^{0}$.

DEFINITION 1. $x^{0}$ is said to be an efficient solution of (VMP) if $x^{0} \in X$ and there exists no $x^{1} \in X$ such that $C x^{1} \geq C x^{0}$.

DEFINITION 2. An efficient solution $x^{0} \in X$ is said to be a properly efficient solution of (VMP) if there exists a scalar $M>0$ such that for each $i \in K, c_{i} x>$ $c_{i} x^{0}$ and $x \in X$ implies that

$$
\left(c_{i} x-c_{i} x^{0}\right) \leqq M\left(c_{j} x^{0}-c_{j} x\right)
$$

for at least one $j(\neq i)$ such that $c_{j} x<c_{j} x^{0}$.

For $x^{0} \in X$, we introduce the following nonlinear program:

$$
\left.\begin{array}{c}
\text { maximize } z=d C x(d>0) \\
\text { subject to } g(x) \leqq 0 \\
-C x+C x^{0} \leqq 0
\end{array}\right\}
$$

where $d \in R^{k}$ is a constant vector.

We shall first relate an efficient solution of (VMP) to an optimal solution of (NLP). This relation is slightly different from the corresponding relations obtained by Isermann [5] and Bhatia and Gupta [2] so that instead of using duality 
theory of nonlinear programming, Kuhn-Tucker conditions can be applied to obtain necessary conditions for an efficient solution of (VMP). This (i) simplifies the approach, (ii) gives necessary conditions in Kuhn and Tucker [9] form and (iii) does not require the convexity of $g$. These conditions are then used to derive a necessary condition for an efficient solution in Isermann [5] form. Also, using a result of Geoffrion [4], we observe that the necessary conditions for an efficient solution are sufficient for a properly efficient solution of (VMP). This leads to sufficient conditions for an efficient solution to be properly efficient. At the end, an example is discussed to illustrate our results.

\section{The results}

LEMMA. $x^{0} \in X$ is an efficient solution of (VMP) if and only if $x^{0}$ is an optimal solution of the nonlinear program (NLP).

Proof. Let $x^{0} \in X$ be an efficient solution of (VMP) and suppose to the contrary, that $x^{0}$ is not an optimal solution of (NLP). Then there exists $x^{*}$ such that

$$
g\left(x^{*}\right) \leqq 0, \quad-C x^{*}+C x^{0} \leqq 0
$$

and $d C x^{*}>d C x^{0}$. Since $d>0$, the above conditions are equivalent to

$$
g\left(x^{*}\right) \leqq 0 \text { and } C x^{*} \geq C x^{0}
$$

which contradicts the fact that $x^{0}$ is an efficient solution (VMP).

Conversely, let $x^{0}$ be an optimal solution of (NLP) and suppose to the contrary, that $x^{0}$ is not an efficient solution of (VMP). Then there exists an $x^{1} \in X$ such that

$$
C x^{1} \geq C x^{0} \quad \text { and } \quad g\left(x^{1}\right) \leqq 0 .
$$

That is, $x^{1}$ is a feasible point of (NLP) and $d C x^{1}>d C x^{0}$ which contradicts that $x^{0}$ is an optimal solution of (NLP). Hence the Lemma.

Whenever we assume that the system (A) satisfies a constraint qualification, we shall mean that (A) satisfies Kuhn-Tucker constraint qualification or weak Arrow-Hurwicz-Uzawa constraint qualification define in Mangasarian [10]. Note that if $g$ is linear, then system (A) always satisfies these constraint qualifications.

The following theorem gives necessary conditions for an efficient solution of (VMP) in Kuhn-Tucker [9] form.

THEOREM 1. Let $x^{0} \in X$ be an efficient solution of (VMP) and let the system (A) satisfy a constraint qualification at $x^{0}$. Then there exist $v^{0} \in R^{k}$ and $w^{0} \in R^{m}$ such that

$$
v^{0} C=w^{0} \nabla g\left(x^{0}\right), \quad w^{0} g\left(x^{0}\right)=0, \quad v^{0}>0, w^{0} \geqq 0 .
$$


Proof. Since $x^{0} \in X$ is an efficient solution of (VMP), by the Lemma, $x^{0}$ is an optimal solution of (NLP). Hence by Theorem 10.2.7 of Mangasarian [10] there exist $w^{0} \in R^{m}$ and $u^{0} \in R^{k}$ such that

$$
\begin{gathered}
-d C+w^{0} \nabla g\left(x^{0}\right)-u^{0} C=0, \\
w^{0} g\left(x^{0}\right)=0, \quad w^{0} \geqq 0, u^{0} \geqq 0 .
\end{gathered}
$$

Since $d>0$, the above conditions imply that

$$
\begin{aligned}
& w^{0} \nabla g\left(x^{0}\right)=v^{0} C, \quad w^{0} g\left(x^{0}\right)=0, \\
& v^{0}=\left(d+u^{0}\right)>0 \quad \text { and } \quad w^{0} \geqq 0 .
\end{aligned}
$$

This proves the theorem.

We now derive necessary conditions for an efficient solution in the form of Isermann [5].

THEOREM 2. Let $x^{0} \in X$ be an efficient solution of (VMP). Let $g_{I}$ be quasiconvex at $x^{0}$ and let the system (A) satisfy a constraint qualification at $x^{0}$. Then there exists $v^{0} \in R^{k}$ with strictly positive components, such that

$$
v^{0} C x^{0} \geqq v^{0} C x \quad \text { for all } x \in X .
$$

Proof. Since $x^{0} \in X$ is an efficient solution of (VMP), by Theorem 1, there exists $v^{0}>0$ and $w^{0} \geqq 0$ such that

$$
\begin{gathered}
v^{0} C=w^{0} \nabla g\left(x^{0}\right), \\
w^{0} g\left(x^{0}\right)=0 .
\end{gathered}
$$

Now let $J=\left\{i: g_{i}\left(x^{0}\right)<0\right\}$. Therefore $I \cup J=\{1,2, \ldots, m\}$. Since $w^{0} \geqq$ $0, g\left(x^{0}\right) \leqq 0$ and $w^{0} g\left(x^{0}\right)=0$, we have that $w_{i}^{0} g_{i}\left(x^{0}\right)=0$ for $i=1,2, \ldots, m$ and hence

$$
w_{J}^{0}=0 .
$$

Now let $x \in X$. Then $g_{I}(x) \leqq 0=g_{I}\left(x^{0}\right)$. Since $g_{I}$ is quasiconvex at $x^{0}$, we have

$$
\nabla g_{I}\left(x^{0}\right)\left(x-x^{0}\right) \leqq 0 .
$$

From (1)

$$
\begin{aligned}
v^{0} C\left(x-x^{0}\right) & =w^{0} \nabla g\left(x^{0}\right)\left(x-x^{0}\right) \\
& =w_{I}^{0} \nabla g_{I}\left(x^{0}\right)\left(x-x^{0}\right)+w_{J}^{0} \nabla g_{J}\left(x^{0}\right)\left(x-x^{0}\right) .
\end{aligned}
$$

Using (3) and (4) we get $v^{0} C\left(x-x^{0}\right) \leqq 0$. Hence $v^{0} C x^{0} \geqq v^{0} C x$ for all $x \in X$. 
THEOREM 3. Let $x^{0} \in X$ and $g_{I}$ be quasiconvex at $x^{0}$. If there exist $v^{0} \in R^{k}$ and $w^{0} \in R^{m}$ satisfying

$$
\begin{gathered}
v^{0} C=w^{0} \nabla g\left(x^{0}\right), \\
w^{0} g\left(x^{0}\right)=0, \\
v^{0}>0, \quad w^{0} \geqq 0,
\end{gathered}
$$

then $x^{0}$ is a properly efficient solution of (VMP).

Proof. Let $v^{0}, w^{0}$ satisfy (5), (6) and (7). Then following the proof of Theorem 2, we get

$$
v^{0} C x^{0} \geqq v^{0} C x \quad \text { for all } x \in X .
$$

Hence by Theorem 1 of Geoffrion [4], $x^{0}$ is a properly efficient solution of (VMP).

The following theorem which gives sufficient conditions for an efficient solution of (VMP) to be properly efficient, follows immediately from Theorems 1 and 3.

THEOREM 4. Let $x^{0} \in X$ be an efficient solution of (VMP). If $g_{I}$ is quasiconvex at $x^{0}$ and the system (A) satisfies a constraint qualification at $x^{0}$, then $x^{0}$ is a properly efficient solution of (VMP).

\section{An example}

Consider the problem

$$
\begin{aligned}
& \operatorname{maximize}\left[c_{1} x=x_{1}, c_{2} x=x_{1}+x_{2}\right]^{T} \\
& \text { subject to } g(x)=x_{1}^{2}+x_{2}^{2}-4 \leqq 0 .
\end{aligned}
$$

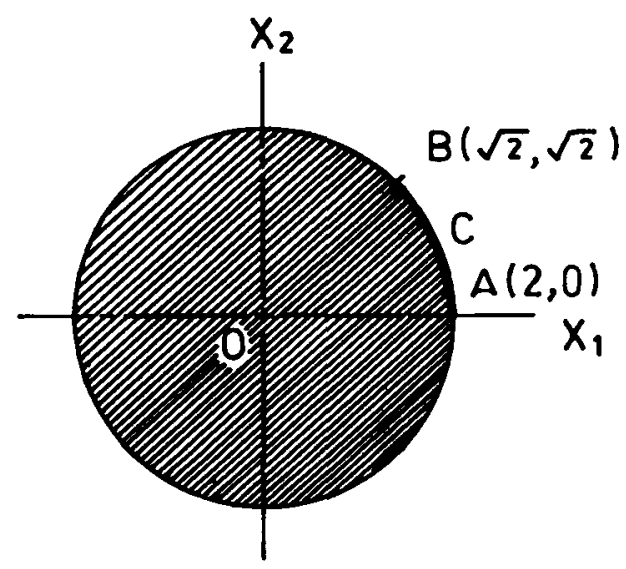

Figure 
The feasible region $X$ is the set of all the points on and inside the circle $x_{1}^{2}+x_{2}^{2}=4$ (the shaded area in the figure). All the points on the circle in the positive quadrant from $A(2,0)$ to $B(\sqrt{2}, \sqrt{2})$ are efficient solutions. The constraint function $g(x)$ is convex on $R^{2}$ and hence quasiconvex.

For an efficient point $x^{0}=\left(x_{1}^{0}, x_{2}^{0}\right)$, the nonlinear program (NLP) is

$$
\begin{gathered}
\text { maximize } z=d_{1} x_{1}+d_{2}\left(x_{1}+x_{2}\right) \\
\text { subject to } x_{1}^{2}+x_{2}^{2}-4 \leqq 0 \\
-x_{1}+x_{1}^{0} \leqq 0 \\
-x_{1}-x_{2}+x_{1}^{0}+x_{2}^{0} \leqq 0 .
\end{gathered}
$$

For each efficient solution $x^{0}$, the above problem does not satisfy the weak ArrowHurwicz-Uzawa constraint qualification. However, it satisfies the Kuhn-Tucker constraint qualification at every point $C\left(x_{1}^{0}, x_{2}^{0}\right)$ on the curve $A B$ except the points $A$ and $B$. Hence, by Theorem 4, all the points on the circle $x_{1}^{2}+x_{2}^{2}=4$ between $A$ and $B$ are properly efficient. That $A$ and $B$ are not properly efficient can be seen from the definition. Consider the point $A$. At this point $x^{0}=(2,0)$, $c_{1} x^{0}=2, c_{2} x^{0}=2$. Also, $c_{1} x^{0}>c_{1} x$ for all $x \in X$ and $c_{2} x>c_{2} x^{0}$ for some $x \in X$. If $x$ approaches to $x^{0}$ along the circle $x_{1}^{2}+x_{2}^{2}=4$, then $c_{2} x>c_{2} x^{0}$, $c_{1} x^{0}>c_{1} x$ and the ratio

$$
\frac{c_{2} x-c_{2} x^{0}}{c_{1} x^{0}-c_{1} x}=\frac{x_{1}+x_{2}-2}{2-x_{1}}=\frac{\sqrt{\left(2+x_{1}\right)}}{\sqrt{\left(2-x_{1}\right)}}-1
$$

can be made arbitrarily large. Hence $A$ is not a properly efficient solution. Similarly, $B$ is also not a properly efficient solution.

Since $g(x)$ is convex on $R^{2}$ we can also apply Theorem 3 to obtain proper efficient solutions without any knowledge of efficient solutions. It says that if for any $x^{0} \in X$, the system

$$
\begin{gathered}
v_{1}+v_{2}=2 w_{1} x_{1}^{0}, \\
v_{2}=2 w_{1} x_{2}^{0}, \\
w_{1}\left(x_{1}^{0^{2}}+x_{2}^{0^{2}}-4\right)=0, \\
v_{1}, v_{2}>0, \quad w_{1} \geqq 0,
\end{gathered}
$$

has a solution, then $x^{0}$ is a properly efficient solution of (VMP). It is very easy to see that the above system is consistent if $x^{0}$ is a point on the circle $x_{1}^{2}+x_{2}^{2}=4$ between $A$ and $B$. At any other point of $X$, the above system becomes inconsistent.

The above discussion also illustrates Theorem 1 . 


\section{Acknowledgement}

The authors are thankful to the University Grants Commission of India for providing financial assistance.

\section{References}

[1] H. P. Benson and T. L. Morin, 'The vector maximization problem: proper efficiency and stability', SIAM J. Appl. Math. 32 (1977), 64-72.

[2] D. Bhatia and B. Gupta, 'Efficiency in certain nonlinear fractional vector maximization problems', Indian J. Pure Appl. Math. 11 (1980), 669-672.

[3] E. U. Choo, 'Proper efficiency and the linear fractional vector maximum problem', Oper. Res. 32 (1984), 216-220.

[4] A. M. Geoffrion, 'Proper efficiency and the theory of vector maximization', J. Math. Anal. Appl. 22 (1968), 618-630.

[5] H. Isermann, 'Proper efficiency and the linear vector maximum problem', Oper. Res. 22 (1974), 189-191.

[6] R. N. Kaul and B. Gupta, 'Efficiency and linear fractional vector maximum problem', $Z$. Angew. Math. Mech. 60 (1980), 112-113.

[7] R. N. Kaul and B. Gupta, 'Multi-objective programming in complex space', Z. Angew Math. Mech 61 (1981), 599-601.

[8] A. Klinger, 'Improper solutions of the vector maximum problem', Oper. Res. 15 (1967), $570-572$.

[9] H. W. Kuhn and A. W. Tucker, 'Nonlinear programming', Proceeding Second Berkeley Symposium on Mathematical Statistics and Probability, pp. 481-492 (Univ. of California Press, Berkeley, California, 1951).

[10] O. L. Mangasarian, Nonlinear programming (McGraw-Hill Inc., New York, 1969).

Department of Mathematics

University of Roorkee

Roorkee 247667

India
Department of Mathematics University of Dhaka

Dhaka-2

Bangladesh 